\title{
An effective method for quality control of the thermocouple
}

\author{
Tingting Wang ${ }^{1}$, Yajun Yan ${ }^{1}$, Yiwen Yuan², Zhuang Ma ${ }^{1}$, Ailiang Zhang ${ }^{3}$, Youyu Chen ${ }^{1}$ and Yimin Yu ${ }^{1}$ \\ ${ }^{1}$ Shanghai Institute of Quality Inspection and Technical Research, Jiangyue Road, Minhang District, Shanghai, China \\ ${ }^{2}$ Shanghai Institute of Special Equipment Inspection and Technical Research, North NujiangRoad, Putuo District, Shanghai, China \\ ${ }^{3}$ Shanghai Institue of Measurement and Testing Technology, Zhangheng Road, New Pudong District, Shanghai, China
}

\begin{abstract}
As a basic testing element, the thermocouples are widely used in the safety and energy efficiency testing of the electronic and electrical products, new energy products and so on. The accuracy of all thermocouples can't be assured for they are mostly calibrated by sampling. In view of this situation, an efficient and convenient method for the intermediate check of the thermocouple is proposed in this paper. Taking 0 degree and 100 degree which can be easily obtained in the nature as the reference standard temperature, the deviation of the testing thermocouples and the reference standard temperature can be determined and the qualification rate of the thermocouples can be obtained. This method can promote the quality control of the laboratory.
\end{abstract}

\section{INTRODUCTION}

Thermocouple is a common temperature measuring element widely used in various temperature tests. The thermocouple is divided into precious metal thermocouple and cheap metal thermocouple, including type B, T, J, K, etc. Although the shape of each type of thermocouple is different, the basic structure and principle is basically the same. The thermocouples are usually made up of the hot electrodes, insulated sleeve protection tubes and the junction boxes, and are usually used together with the display instruments, the recording instruments and the electronic adjusters. The thermocouples do not measure the temperature directly, but rather an energy converter that converts heat energy into the electrical power, which is generally called thermoelectric potential using the Seebeck effect.

The safety and energy efficiency tests for electronic appliances and new energy products need thermocouples. The electrical safety test, such as temperature rise test, measure the surface or internal temperature of the sample under normal working or failure conditions. When the temperature exceeds a certain limit, the product can easily cause overheating, spontaneous combustion and other safety accidents. Energy efficiency tests for electric appliances such as the kitchen appliances also require the use of thermocouples, which directly assess the heating of the appliance. It can be seen from the above that, the accuracy of the thermocouple is very important, which affects the test results directly.

An efficient and convenient method for the intermediate check of the thermocouple is proposed in this paper. The deviation of the measured thermocouple and the reference standard temperature can be determined and the qualification rate of the thermocouples can be obtained. This method can be used to evaluate the quality of the thermocouples in a simple and efficient way.

\section{CHECK PROCEDURE OF THE THERMOCOUPLES}

As an important test element, the quality of the thermocouples should be controlled. The accuracy of the thermocouples should meet the requirements of JJF 1637-2017 "The calibration specification of the cheap metal thermocouple ", JJG 141-2000 "Precious metal thermocouple verification procedure" and so on. When the material of the thermocouple is uniform, the thermoelectric potential has nothing to do with the length and diameter of the thermocouple, which is only related to the composition of the thermocouple material and the temperature difference between the two ends. Considering this characteristic of thermocouple, most laboratories calibrate the thermocouples by the random sampling method.

However, the sampling selection must have a leak detection. It can only ensure the accuracy of the calibrated thermocouples but not all the using thermocouples. Here, a simple and efficient quality control method is proposed.

The check procedure consists of two parts, the testing in ice water mixture and boiling water, which are easily obtained in the natural. Check the deviation of the value of the testing thermocouples at freezing point and boiling point with the reference standard temperature. If the

\footnotetext{
* Corresponding author: wangtt@ sqi.org.cn
} 
deviation is within the standard limit value scope, it is considered that the check result is passed.

The procedure is as follows:

- Fill the ice water mixture to the container and stir well to ensure the temperature is uniform. If available, use platinum resistance or standard mercury thermometer to determine the temperature uniformity of each point;

- The thermocouples are connected to the data acquisition instrument and record the channel of each thermocouple;

- Put the thermocouples into the ice water mixture at the same time. The data acquisition instrument will read the data and save it;

- Fill the boiling water to the container and stir well to ensure the temperature is uniform. If available, use platinum resistance or standard mercury thermometer to determine the temperature uniformity of each point;

- Put the thermocouples into the boiling water at the same time. The data acquisition instrument will read the data and save it;

- Draw the numerical curves. Calculate the average of the temperature of different channel at 0 and 100 degree for a period of testing time. Calculate the deviation between the value and the standard reference temperature and determine whether the deviation exceeds the standard limit value. If not exceed, the thermocouple check is qualified. If exceed, the thermocouple check is not qualified;

- If the verification result is abnormal, the reason shall be analyzed and the calibration shall be confirmed. If there is a problem with the quality of the thermocouple, it should be replaced in time so it will not affect the test result.

Also, the following points should be noted in the test:

- Pay attention to the correctness of the welding of thermocouple end points;

- The thermocouple should not be arranged on the edge of the container to avoid the error caused by the edge temperature.

- Multiple checks can be made to avoid accidental mistakes.

- Make reasonable check frequency and ensure meet laboratory's usage requirements.

\section{CHECK RESULTS OF THE THERMOCOUPLES}

In this paper, T-type (copper-constantan) thermocouple is used as the testing samples.

Test conditions: Temperature: 17.6 degree; humidity: 46.7\%RH; Atmospheric pressure: 101.72kpa.

Test equipment: Aglient 34970A.

Fig. 1 and Fig. 2 show the values of 8 thermocouples collected by the data acquisition instrument in 0 degree (ice water mixture environment) and 100 degree (boiling water environment). The $\mathrm{X}$-axis is the time and the unit is second. The $\mathrm{Y}$-axis is the temperature and the unit is Celsius. The collection time is 600s.
It can be seen from the figures that in the time of $600 \mathrm{~s}$, the collection data of thermocouple is relatively stable. There are slight fluctuations in the diagrams, which may be influenced by small factors such as ambient temperature and wind speed.

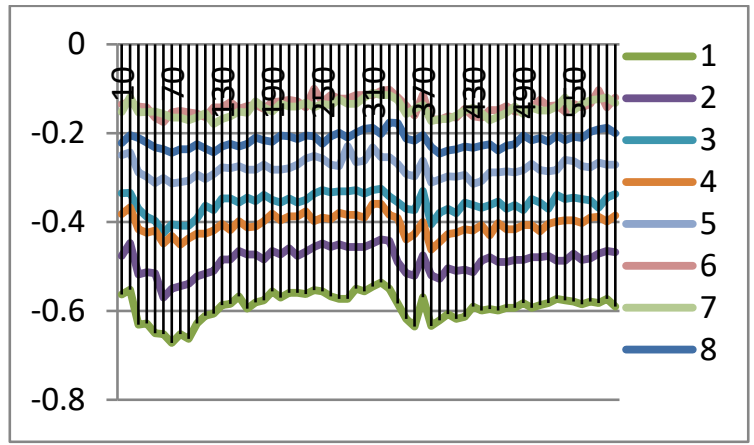

Figure 1: The values of different thermocouples in 0 degree

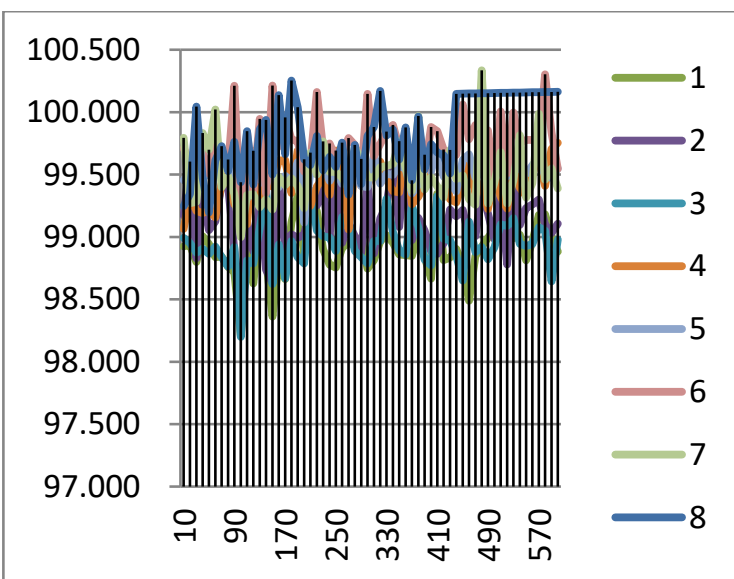

Figure 2: The values of different thermocouples in 100 degree .

Then the averages $(\overline{\mathbf{T}})$ of the 8 thermocouples at 0 and 100 degrees can be gotten. Compare the average with the reference standard temperature. And a deviation $(\Delta \mathbf{T})$ can be obtained. The deviation in 0 degree is $\Delta \mathrm{T}=\left|\overline{\mathrm{T}_{0}}\right|$. The deviation in 100 degrees is $\Delta \mathrm{T}=\left|100-\overline{\mathrm{T}_{100}}\right|$.

The testing thermocouples are T-type of class II. According to JJF 1637-2017 "The calibration specification. of the cheap metal thermocouple ", the allowable deviation of the T-type thermocouple (class II) is 1 degree.

From table 1 and table 2, it can be obtained that:

- The test value of channel 1 and channel 3 at 100 degrees is lower than the reference standard temperature. The deviation was 1.095 and 1.098 respectively, exceeding 1 degree. So the intermediate check of the channel 1 and channel 3 are not passed. Repeated measurement can be used to confirm the qualification of the thermocouples;

- According to the test results in 0 and 100 degrees, the qualified rate was $75 \%$. Therefore, it is necessary and meaningful to check the thermocouple periodically. 
Table 1: The averages and deviations of data collected by different thermocouples in 0 degree (unit: ${ }^{\circ} \mathrm{C}$ ).

\begin{tabular}{|c|c|c|}
\hline channel & channel 1 & channel 2 \\
\hline average $\overline{\mathbf{T}_{\mathbf{0}}}$ & -0.590 & -0.486 \\
\hline deviation $\Delta \mathbf{T}$ & 0.590 & 0.486 \\
\hline channel & channel 3 & channel 4 \\
\hline average $\overline{\mathbf{T}_{\mathbf{0}}}$ & -0.358 & -0.406 \\
\hline deviation $\Delta \mathbf{T}$ & 0.358 & 0.406 \\
\hline channel & channel 5 & channel 6 \\
\hline average $\overline{\mathbf{T}_{\mathbf{0}}}$ & -0.280 & -0.138 \\
\hline deviation $\Delta \mathbf{T}$ & 0.280 & 0.138 \\
\hline channel & channel 7 & channel 8 \\
\hline average $\overline{\mathbf{T}_{\mathbf{0}}}$ & -0.145 & -0.216 \\
\hline deviation $\Delta \mathbf{T}$ & 0.145 & 0.216 \\
\hline
\end{tabular}

Table 2: The averages and deviations of data collected by different thermocouple in100 degree (unit: ${ }^{\circ} \mathrm{C}$ ).

\begin{tabular}{|c|c|c|}
\hline channel & channel 1 & channel 2 \\
\hline average $\overline{\mathbf{T}}_{\mathbf{1 0 0}}$ & 98.905 & 99.124 \\
\hline deviation $\Delta \mathbf{T}$ & $1.095^{*}$ & 0.876 \\
\hline channel & channel 3 & channel 4 \\
\hline average $\overline{\mathbf{T}} \mathbf{1 0 0}_{\mathbf{1}}$ & 98.942 & 99.428 \\
\hline deviation $\Delta \mathbf{T}$ & $1.058^{*}$ & 0.572 \\
\hline channel & channel 5 & channel 6 \\
\hline average $\overline{\mathbf{T}}_{\mathbf{1 0 0}}$ & 99.501 & 99.745 \\
\hline deviation $\Delta \mathbf{T}$ & 0.499 & 0.255 \\
\hline channel & channel 7 & channel 8 \\
\hline average $\overline{\mathbf{T}_{\mathbf{1 0 0}}}$ & 99.504 & 99.821 \\
\hline deviation $\Delta \mathbf{T}$ & 0.496 & 0.179 \\
\hline
\end{tabular}

\section{CONCLUSIONS}

The accuracy of all thermocouples can't be assured for they are be calibrated by sampling in mostly in the laboratory. In view of this situation, an efficient and convenient method for the intermediate check of the thermocouple is proposed in this paper. Taking the 0 degree and 100 degrees which can be easily obtained in the nature as the reference standard temperature, the deviation of the testing thermocouple and the standard temperature can be determined and the qualification rate of the thermocouples can be obtained. This check method is simple and quick, easy access to test environment. Also, this method has high efficiency for the channel of the data acquisition instrument can reach 60 or more. The thermocouples can be tested in batches.

\section{ACKNOWLEDGEMENTS}

This paper is funded by the project " 2017 QK020 Research and evaluation on safety and performance of the hydrogen storage bottle for advanced energy vehicles." by general administration of quality supervision inspection and quarantine of people's republic of china and "17DZ1200800 Research on hydrogen cycle test technology for hydrogen storage bottle in fuel cell vehicle (phase I) " by shanghai science and technology commission.

\section{REFERENCES}

1. Jianlin, T., 2012. The application of the intermediate checks in testing and calibration institutions, Calibration and testing techniques, 39(4).

2. Ling X., 2009. Brief Introduction on Intermediate Check to First-Class Standard Platinum and Rhodium (10)-Platinum Thermocouple, Jiangsu electrical engineering, 6.

3. Rybakov Y.V., 1991,Investigation of intermediate process of thermocouple cable rolling, ResearchGate.

4. Jahan F., Ballico M.J., 2013. Traceable Co-C eutectic points for thermocouple calibration, ResearchGate .

5. JJF 1637, 2017. The calibration specification of the cheap metal thermocouple, general administration of quality supervision inspection and quarantine of people's republic of china. China, $1^{\text {st }}$ edition.

6. JJG 141, 2000, Precious metal thermocouple verification procedure. general administration of quality supervision inspection and quarantine of people's republic of china. China, $1^{\text {st }}$ edition. 